\title{
Análisis de costos en evaluaciones económicas en salud: Aspectos introductorios
}

\author{
RONY LENZ-ALCAYAGA ${ }^{1, a}$
}

'Escuela de Salud Pública, Universidad de Chile. ${ }^{a}$ Economista.

Correspondencia a: Rony Lenz-Alcayaga Jorge Matte Gormaz 1390 Providencia, Santiago de Chile. Teléfono/fax: 029812144 E-mail: rlenz@lenz.cl

\section{Cost analysis in economic evaluation of healthcare: An introduction}

Cost analysis is a central part of economic appraisal in healthcare and a constant worrying for planners and assessment teams, despite its complexity and the difficulty of making estimates. Most important economic terms related to cost analysis are reviewed. Economics common idea of cost, centered in resource allocation, is the concept of opportunity cost or the sacrifice that is made when an alternative is chosen, expressed in terms of the one that was rejected. In every economic assessment relevant cost must be identified as well as the perspective of costing. Complexity of costing analysis can be reduced by an appropriate definition of the objectives of cost, a detailed description of the process associated to the health intervention, and considering identification, quantification and valuation of the resources consumed by an intervention.

(Rev Med Chile 2010; 138 (Supl 2): 88-92).

Key words: Costs, cost analysis, economic evaluation.

\section{RESUMEN}

El análisis de costos es parte central de la evaluación económica en salud y una preocupación de evaluadores y planificadores, a pesar de su complejidad. En este trabajo se revisan los principales términos económicos asociados al análisis de costos. La noción de costos generalizada en economía, cuyo propósito es apoyar la asignación de recursos, enfatiza en concepto de costo de oportunidad, esto es, el sacrificio en que se incurre al optar por una alternativa, el cual está dado por la alternativa que se abandona. En toda evaluación hay que identificar los costos que son relevantes y la perspectiva de la evaluación. La complejidad inherente al problema del costeo puede reducirse definiendo correctamente el objeto del costeo, realizando una descripción detallada del proceso asociado al servicio que se quiere costear y considerando otros aspectos críticos como el identificar, cuantificar y valorizar el consumo de recursos asociados a las intervenciones.

Palabras clave: Costos, análisis de costos, evaluación económica
L a evaluación económica corresponde al análisis comparativo de las acciones alternativas en términos de sus costos y consecuencias ${ }^{1,2}$. El análisis de costos es parte central de la evaluación y una preocupación de los evaluadores ${ }^{3}$. La complejidad creciente en la selección de procedimientos diagnósticos y terapéuticos, la amplia gama de medicamentos, la necesidad de reestructurar la oferta de servicios, el surgimiento de pandemias, etc., son ejemplos de la necesidad de 
evaluar alternativas y analizar costos. Este trabajo revisa los principales conceptos del análisis de costos y enfatiza su complejidad, las dificultades en su cálculo y los desafíos que enfrentan los evaluadores.

\section{El concepto de costo}

La economía coloca el acento en la asignación recursos, enfatizando el concepto de costo de oportunidad, esto es, el sacrificio en que se incurre al optar por una alternativa, expresado en términos de la mejor alternativa abandonada. El costo de oportunidad señala que los costos están relacionados con los procesos de elección ${ }^{4}$. Los costos son relativos y no absolutos. Los productos, recursos o proyectos tienen costos en función de circunstancias específicas y de quien esté tomando las decisiones. Los costos pertinentes para una decisión son los costos relevantes.

Los costos relevantes a considerar dependen de la perspectiva que asume la evaluación. Los pacientes, prestadores y financistas, tienen perspectivas distintas, porque trasladan costos de forma diferente. Para un prestador los costos directos son los relevantes. El paciente, dado que los seguros financian la atención, considera lo contrario. Los costos directos, se asocian con los recursos propios de los sistemas de salud, mientras que los costos indirectos se relacionan con el valor del tiempo que tiene que invertir el paciente en una intervención (traslado, espera, recuperación, etc.), asociado al salario y a su productividad. La economía, recomienda adoptar una perspectiva más amplia: la perspectiva social. El costo social se refiere a los costos que afectan a todos y cada uno de los miembros de la sociedad.

El costo corresponde al valor del consumo de recursos en que se incurre para generar un servicio, el cual puede ser económico o financiero. Cuando es financiero, el costo implica un desembolso monetario, cosa que no necesariamente ocurre cuando es económico. Los costos tienen dos componentes: el consumo físico de recursos asociado al proceso de provisión y la asignación de un valor a este consumo físico. Cuando expresamos el consumo de recursos en términos unitarios podemos hablar de precio.

El precio que habría que utilizar para valorar los recursos es el costo de oportunidad. Técnicamente, su cálculo implica conocer la función de bienestar social, cosa que la economía no ha podido realizar 5 . Bajo ciertos supuestos -equilibrio competitivo y aceptar la distribución de ingresos- los precios de mercado de los recursos son buenos estimadores de los costos de oportunidad. En los sistemas de salud los precios no son buenos estimadores: Están distorsionados por fallas de mercado, existen terceros pagadores $\mathrm{y} / \mathrm{o}$ intervención estatal; desequilibrio o inexistencia de mercados; $y$, precios inestables e impredecibles.

El concepto de costo de oportunidad, presenta dificultades prácticas al realizar estudios empíricos. Los datos disponibles se refieren a costos contables. La contabilidad, cuya función es alimentar la planeación y el control financiero, hace énfasis en los registros histórico o costo histórico. El análisis de costos adapta los costos contables al concepto de costos de oportunidad. Esto explica su complejidad.

El análisis microeconómico, ayuda a entender y diferenciar el comportamiento de los costos. Los costos totales, consideran el total de recursos que se consumen para generar la producción. Pueden descomponerse según el tipo de recursos productivo (trabajo, insumos, capital, etc) o según su asociación con el volumen de producción. Los costos fijos no varían con esta última. Los costos de capital-las camas, equipamiento, administración, etc-son costos fijos. Los costos variables -trabajo, insumos, medicamentos, etc-, están relacionados con los niveles de producción. Esta distinción entre costos fijos y variables, es válida mientras no se altere la capacidad productiva total de la unidad. Esto se denomina corto plazo y no está asociado a un período temporal específico. En un horizonte que permita el redimensionamiento total de la actividad -conocido como largo plazo-, todos los recursos son variables.

Los costos totales y costos unitarios están asociados. Los costos unitarios se refieren al costo de producir una unidad, entre ellos los costos medios y los marginales. En el corto plazo, los costos unitarios son directamente proporcionales al pago efectuado para disponer del recurso e inversamente proporcionales a su productividad. Así, para un salario dado, mientras mayor sea la productividad del trabajo -el principal recurso de los sistemas de salud-menor es el costo unitario de producción. Esta es la "ley de rendimientos decrecientes al factor" e indica que la productividad de los recursos variables es decreciente. 
Microeconómicamente también se considera los costos a largo plazo, es decir, cuando todos los factores son variables, incluyendo el stock de capital. Se postula que los costos medios y los costos marginales de largo plazo también tienen de forma de "U" pero sobre un rango de producción mucho más amplio. La explicación está dada, porque existen economías y deseconomías de escala.

\section{Estimación de costos para las evaluaciones económicas}

Los costos de una intervención en una evaluación económica se denominan costos incrementales, es decir, en los cuales habría que incurrir si se lleva a cabo la intervención. De otra manera, no se habrían realizado y por tanto, son directamente atribuibles a esta. Cuando la comparación de costos está relacionada con intervenciones restringidas a un solo problema, no es necesario considerar todos los costos asociados, sino tan sólo aquellos en que las intervenciones difieren. Por ejemplo, si estamos evaluando la costo-efectividad de un medicamento oncológico, complementario al tratamiento base, bastará considerar los costos incrementales, es decir, principalmente el medicamento y los costos netos que induce. Su ventaja es reducir el trabajo de análisis de costos, sin afectar la calidad de la evaluación. Si se espera que los antecedentes sirvan para una comparación más amplia, incluyendo alternativas no especificadas, es conveniente incluir todos los costos.

La estimación de costos supone, además de definir correctamente el problema que se quiere abordar -objeto del costeo- y una descripción detallada del proceso asociado al servicio que se quiere costear, otras tres etapas: Identificar el consumo de recursos asociados a las intervenciones; cuantificar el consumo físico de recursos; $y$, valorizar el consumo de recursos.

\section{Identificación del consumo de recursos}

En la identificación del uso de recursos es conveniente distinguir: i) Recursos de atención (médicos, insumos, fármacos, etc) requeridos directamente en la intervención; ii) Recursos de atención que en el futuro son inducidos o son evitados por la intervención; iii) Tiempo del paciente incluyendo los cambios en la productividad; y, iv) Tiempo de cuidadores del paciente y costos informales asociados. Dependiendo de la perspectiva de la evaluación uno u otro componente podrán incorporarse, incluyendo todos en la perspectiva social.

Es importante el horizonte temporal de la evaluación: El período donde la intervención genera efectos. En la medida que las intervenciones no alteren sustantivamente la capacidad de producción y no impliquen la posibilidad de sustituciones importantes entre factores -por ejemplo, entre trabajo y capital- es aceptable trabajar con costos marginales de corto plazo.

\section{Cuantificación del consumo de recursos}

La cuantificación del uso de recursos puede ser prospectiva o retrospectiva. Bajo la primera, se preparan, como parte de los estudios clínicos aleatorizados, los formularios que recabarán información de consumo de recursos asociados a los protocolos de atención. El retrospectivo -usar las fuentes de registro rutinario de los establecimientos de salud-, es el más utilizado. El esfuerzo necesario para estimar los costos debe ser proporcional a la importancia de los mismos. No conviene invertir tiempo y recursos en costos que son irrelevantes en la decisión.

La base de cálculo del consumo de recursos puede tener distinto nivel de detalle o bases de costeo. En el extremo, el método que entrega mayor precisión, está el costeo de cada una de las atenciones, denominado micro-costeo. Se identifican actividades (consulta médica, los exámenes de apoyo diagnóstico, los días cama, etc) para cada una de las fases de la intervención para cada tipo de paciente.

El costeo sobre la base de una canasta estandarizada de prestaciones, tipo GRD, en el cual se toma el costo de resolución de un caso para un paciente promedio. En este caso, no es necesario identificar cada una de las actividades que involucra la intervención, sino sólo el conjunto estandarizado de intervenciones que supone la resolución del problema.

Menos preciso es considerar el costo promedio diario asociado al tratamiento en cada categoría de enfermedad. Este método corresponde a un híbrido entre el método anterior y el método si- 
guiente, donde se realiza un esfuerzo por asociar el consumo de recursos a un tipo de paciente.

Finalmente, el método más impreciso es tomar el valor que comúnmente está disponible en los sistemas de salud, es decir, el costo promedio diario de atención sobre todas las categorías de pacientes, como por ejemplo, el valor del egreso medio.

Este último corresponde al sistema que se ha ido popularizando y cuya base de estimación corresponde al método de costeo por escalonamiento o cascada. Bajo este método, el establecimiento de salud se organiza bajo tres tipos de centros de costos: finales, intermedios y de apoyo. Los finales son los que entregan atenciones directas al paciente. Los intermedios generan servicios de apoyo diagnóstico y terapéutico. Los de apoyo entregan soporte logístico y administrativo. Los costos se organizan en directos - propios de cada centro de costos-y los indirectos -aquellos que provienen de otros centros-. Luego se trasladan los costos de los centros de apoyo a los intermedios en base a parámetros físicos de servicios y posteriormente de los centros intermedios a los finales.

Otro problema es el del tratamiento que debe dársele al consumo del capital. Un bien de capital, por ejemplo un equipamiento médico, no se consume en un período, sino que su vida útil y su capacidad productiva se extienden en el tiempo. Para entender el consumo de capital hay que distinguir entre la propiedad del bien de capital y su uso. Si no hay necesidad de adquirirlo para usarlo, entonces económicamente habría que arrendarlo. Por diversas razones, entre ellas disminuir el riesgo, los bienes de capital son adquiridos y por ende el cálculo del consumo debe realizarse sobre el valor de adquisición del bien. Esto obliga a asociar aquella parte del bien de capital que es consumido por unidad de tiempo a través de un procedimiento que se conoce como anualización de los gastos de capital.

\section{Valorización del consumo de recursos}

La valorización consiste en determinar el "precio" que hay que pagar por los recursos, tomando en cuenta la perspectiva de la evaluación. Los financistas optan por ponderar las atenciones por los precios de transferencia que utilizan para financiar las actividades de salud. Esto se fundamenta, en que los seguros pueden acceder a estas actividades a través de diversos prestadores.

Lo anterior no coincide con la perspectiva del prestador, el cual incurre en costos para proveer la intervención que pueden estar por sobre o bajo el precio del seguro. Dado su mayor tamaño financiero, los seguros imponen precios "monopsónicos", menores a los que resultarían de un equilibrio competitivo. Desde una perspectiva social, el problema es complejo y no tiene fácil solución.

Los costos no se incurren en un mismo momento, sino que se distribuyen en el tiempo. La sociedad no valora igual disponer de recursos para el consumo hoy, que disponer de los mismos en el futuro. Esto se conoce como preferencia intertemporal. Esta está sesgada hacia una mayor valoración de los sucesos que ocurren en el presente. La medida en que la sociedad está dispuesta a sacrificar consumo presente por consumo futuro se conoce como tasa de preferencia intertemporal y sirve para darle valor al desplazamiento de recursos de un período a otro.

En mercados de capitales perfectos -donde existe un arbitraje perfecto y certidumbre- la tasa de interés de mercado refleja esta tasa de preferencia. Dado que estos mercados no son perfectos, es necesario recalcular la tasa de preferencia intertemporal a partir de la tasa de interés, de manera de obtener la tasa de descuento social para las evaluaciones económicas. En Chile, este cálculo lo realiza MIDEPLAN como parte del Sistema Nacional de Inversiones. Para el año 2009 esta tasa se estimó en 6,0\% y permite calcular los factores de descuento. El factor de descuento cuando la tasa de descuento es constante, corresponde a $1 /(1+\mathrm{i})$ ${ }^{\mathrm{n}}$ para cada año (donde $\mathrm{n}$ es el año e i es la tasa de descuento social). Así, en el presente (año 0) el factor es 1, mientras que más nos alejamos en el futuro el factor desciende.

\section{Conclusión}

El análisis de costos es esencial en las evaluaciones económicas. El problema particular que se trata de dilucidar y la perspectiva de la evaluación, condicionan la metodología. La complejidad inherente al costeo se reduce, si se definir correctamente el objeto del costeo. Identificación, cuantificación y valoración del consumo de recursos son claves. La escasa disponibilidad de información coloca límites y obliga al evaluador 
a realizar concesiones juiciosas que no desvirtúen el propósito de la evaluación.

\section{Referencias}

1. Weinstein MC, Stason WB. Foundations of cost-effectiveness analysis for health and medical practices, New England Journal of Medicine 1977; 296: 716-21.

2. Drummond m, O’Brien b, Stoddart G, Torrance G. Mé- todos para la Evaluación Económica de los Programas de Asistencia Sanitaria. Segunda Edición, originalmente publicado en 1997. Ediciones Díaz Santos S.A. Madrid.

3. Gardner M. Cost Analysis in Obstetrics and Gynecology. Clinical Obstetrics and Gynecology 1998; 41-2: 296-306.

4. Buchanan J. Cost and Choice: An Inquiry in Economic Theory. Liberty Fund, Inc. Indiana. 1969.

5. Mogyorosy Z, Smith P. The Main Methological Issues in Costing Health Care Services, a Literature Review. CHE Research Paper, York. 2005. 